\title{
Philosophiques
}

Piotte, Jean-Marc, Marxisme et pays socialistes, Montréal, VLB éditeur, 1979, 177 p.

\section{Laurent-Michel Vacher}

Volume 6, numéro 2, octobre 1979

URI : https://id.erudit.org/iderudit/203122ar

DOI : https://doi.org/10.7202/203122ar

Aller au sommaire du numéro

Éditeur(s)

Société de philosophie du Québec

ISSN

0316-2923 (imprimé)

1492-1391 (numérique)

Découvrir la revue

Citer cet article

Vacher, L.-M. (1979). Piotte, Jean-Marc, Marxisme et pays socialistes, Montréal, VLB éditeur, 1979, 177 p. Philosophiques, 6(2), 309-323.

https://doi.org/10.7202/203122ar d'utilisation que vous pouvez consulter en ligne.

https://apropos.erudit.org/fr/usagers/politique-dutilisation/ 
PIOTTE, Jean-Marc, Marxisme et pays socialistes, Montréal, VLB éditeur, 1979, $177 \mathrm{p}$.

par Laurent-Michel Vacher

Ce livre n'est pas d'un abord facile dans la mesure où son objet, sa méthode et ses thèses ne se dégagent que progressivement et jamais de façon univoque, claire ni systématique. Pour ce motif, le présent compte rendu procédera à un examen partie par partie.

Dans une première section de son introduction intitulée D'où j'écris, l'auteur part (sans aucune mise en situation préalable) des « Nouveaux Philosophes » français. Il s'en prend à eux avec virulence: conception idéaliste de l'histoire, réduction des réalités complexes du monde soviétique au seul Goulag, amalgames philosophiques injustifiés ${ }^{1}$, pauvreté de leur conception d'un "Pouvoir " inamovible et d'une "Résistance " éthique, plébéienne et artistique, sophismes et paradoxes, etc. Il nous suggère quelles sont selon lui les bases et les fonctions historiques et sociales de cette "école ": désillusion de la génération intellectuelle de 1968 ; exploitation (d'ailleurs non décisive) par la droite et les média dans la bataille électorale en France contre l'Union de la Gauche et dans la promotion internationale du libéralisme entreprise par la Trilatérale.

On peut relever deux détails significatifs dans ces quelques pages. J.-M. Piorte semble attaquer sur la base d'une position de type marxiste (conception matérialiste de l'histoire, rôle essentiel des luttes de classes). Par ailleurs, il écrit : les sociétés «socialistes» (avec guillemets manifestement dépréciatifs), le pouvoir "socialiste », et parle sans réserve apparente

1. Chez Glucksmann, entre Fichte, Hegel, Marx et Nietzsche. 
de la "bureaucratisation du socialisme", des "déformations des pays socialistes", de la nécessité de "reconnaître et d'analyser les phénomènes qui contredisent le caractère socialiste des pays ainsi qualifiés »(p. 16) ${ }^{2}$.

Le sens de l'attaque contre Lévy, Glucksmann et Cie semble donc le suivant : J.-M. Piotte veut nous informer à la fois qu'il est loin d'être l'un d'eux (étant marxiste) et qu'il est prêt à faire siennes certaines des questions qu'ils soulèvent (et que les «courants marxistes dominants » ont eu le tort de négliger) ainsi que certains aspects peut-être de leurs réponses. Le problème principal qui se dégage est finalement le suivant : quelle est la nature réelle des pays « socialistes"? L'auteur rejette brièvement les réponses qu'y donnent traditionnellement le Parti communiste français, puis J. Ellenstein, L. Althusser, Ch. Bettelheim et les trotskystes.

À partir de là, dans une seconde partie de son introduction, il annonce le déroulement d'ensemble de son ouvrage. Il pose, en guise de postulat, que "le marxisme demeure la théorie qui nous permet le mieux de comprendre et d'expliquer le capitalisme » et qu'à ce titre on devrait «utiliser les connaissances marxistes pour comprendre et expliquer ce qu'il est advenu du "socialisme" "(p. 18). Pour lui, cela implique qu'on soit prêt à mettre en question, à corriger, développer ou réviser le marxisme, à la lumière des données historiques, pour « rendre compte de phénomènes que n'avait pas prévus Marx ou n'avait pas compris Lénine » (ibid.). Ceci suppose d'après lui une critique de l'opposition science/idéologie (chapitre 1) : le marxisme est-il scientifique? La distinction science/idéologie est-elle fondée et quels en sont les effets politiques? L'auteur nous annonce alors que, de ses discussions sur ces questions, il tirera "trois postulats": "primauté de la matière sur l'esprit; tout phénomène social doit être compris dans son historicité, dans ses contradictions ; celles-ci sont structurées par la lutte de classes» (p. 18). Il abordera ensuite la question des rapports parti/classe (deuxième chapi-

2. On chercherait en vain dans la suite du livre une liste de ces phénomènes ou une quelconque définition de ce qui constituerait, selon J.-M. Piotte, le " caractère socialiste "d'une société. 
tre), le développement du contrôle bureaucratique ( $\mathrm{ch}$. 3) et la nature de l'État (ch. 4), la question des nationalités (ch. 5), la nature des sociétés "socialistes" (ch. 6), les relations des pays de l'Est avec l'extérieur et le conflit bureaucratie/bourgeoisie à l'échelle mondiale (ch. 7) et, enfin, l'enjeu historique des luttes actuelles (ch. 8).

De même qu'il a explicité au passage les trois " postulats " de sa philosophie de base (au sujet desquels, assez incroyablement, nous n'en apprendrons guère plus), J.-M. Piotte énonce dans cette brève présentation quelques-unes de ses thèses principales: responsabilité de Lénine dans la bureaucratisation ; irréductibilité de l'oppression des nationalités (dominées par la bureaucratie russe) à la lutte des classes ; définition des sociétés "socialistes" comme un type nouveau et original de société de classes où une domination de nature plus politique qu'économique est exercée par une classe bureaucratique; opposition entre " marxistes marginaux" (parmi lesquels il se range) et " marxisme dominant ".

Muni de cette vue d'ensemble, le lecteur peut aborder le premier chapitre comme un bon exemple de la "méthode " de l'auteur. De courts commentaires de textes passent en revue les points de vue successifs de Marx (dont la justesse et la richesse sont soulignées), d'Engels (qui durcit les thèses du précédent) ${ }^{3}$, de Lénine (qui aggrave le durcissement), de Staline (qui sombre dans le dogmatisme), puis de Gramsci (qui nous indique les voies d'une solution $)^{4}$. En deux pages, Piotte conclut alors en énonçant sa propre thèse, souvent sans que le lien avec les discussions et les citations antérieures apparaisse clairement sur l'essentiel. Dans le cas présent, cette conclusion est que : 1) le marxisme est un savoir complexe, ayant à la fois des aspects scientifiques et des composantes idéologiques (éthiques, politiques); 2) le matérialisme (primat de la matière sur l'esprit) ne peut être qu'un postulat philosophique indémontrable, non une vérité scientifique. Dans un raccourci saisissant (et répétitif) il ajoute: « Je retiendrai aussi du

3. Dans d'autres chapitres, c'est de Marx et Engels ensemble que la souplesse sera opposée aux thèses rigides de Lénine.

4. Selon les chapitres, tel auteur pourra être passé sous silence ou tel autre venir s'intercaler (Kautsky, Rosa Luxembourg, Mao, Althusser, Ch. Berrelheim), sans toutefois changer ce "pattern " uniforme. 
marxisme deux énoncés que je poserai comme postulats : tout phénomène social (économique, politique et idéologique) doit être compris dans son historicité, dans ses contradictions; la lutte des classes structure celles-ci » (p. 33).

L'une des choses surprenantes qui se confirment dans ce chapitre, c'est que l'auteur aime procéder davantage par affirmation péremptoire que par argumentation. Ainsi, des quatre thèses rappelées ci-dessus, une seule est développée (que le marxisme ne soit pas une science) et cela uniquement sur la base du jugement selon lequel «il est structuré par des valeurs et un espoir » et de la proposition (elle-même non expliquée ni démontrée) selon laquelle "l'idéologie est inhérente à toute pensée politique $»($ p. 32), (ce qui revient à s'accorder ce qui était précisément en question). Les trois autres conclusions sont: l'une (que le matérialisme soit un postulat) appuyée seulement sur une longue citation de Gramsci dont il est loin d'être évident qu'elle aille dans le même sens; les deux autres énoncées sans rapport avec ce qui précède (ces énoncés n'ont pas évolué depuis l'introduction : leur forme est la même et ils ne sont pas davantage justifiés; nous ignorerons également ce que l'auteur rejette du marxisme et pourquoi). Mon lecteur pensera peut-être que j'exagère, mais je crois qu'on peut affirmer que, chez J.-M. Piotte, l'importance d'une thèse paraît en rapport inverse avec le souci qu'il a de l'expliquer et de la justifier.

Naturellement, un certain malaise ne peut ici manquer de se faire jour. Dans une introduction intitulée D'où j'écris, un auteur qui se prétend marxiste ne nous apprend à peu près rien sur ses positions politiques passées ou présentes ni sur les raisons qui le conduisent, au Québec en 1979, à traiter de ces questions. On ne sait vraiment guère d'où il écrit. Dans un texte intitulé Questions de philosopbie, il associe "philosophique " à "indémontrable " et ramène sa pensée à quatre brefs "postulats" qui supposent réglées en une couple de phrases à l'emporte-pièce quelques-unes des questions les plus difficiles de la pensée marxiste débattues par des centaines de théoriciens depuis un siècle. La suite du livre malheureusement n'arrangera rien. 
Le second chapitre, sur la dialectique classe/parti, se présente lui aussi comme un panorama de positions d'auteurs classiques, sous forme d'une succession de commentaires de textes et de citations. Le but semble être le suivant : montrer que Lénine a, en la matière, déformé l'inspiration marxiste originelle. Bien que de courts paragraphes fassent ici et là allusion aux grandes lignes du contexte historique (p. 46-7 pour la Russie, p. 49 pour la Chine), cette mise en situation ne donne pas lieu à une analyse en termes de classe des idéologies en présence. Aucune conclusion autre que "la nécessaire activité des masses pour réaliser la révolution" (p. 53) n'est explicitée. L'auteur note bien au passage : " La composition de ces partis peut varier (paysanne, ouvrière ou autre), mais l'origine de classe de sa direction (sic) demeure toujours identique : petite-bourgeoise $\gg$ (p. 54). Mais ceci ne va pas bien loin, sous la plume de quelqu'un qui nous a avertis quelques pages auparavant que «l'origine de classe peut parfois éclairer, mais n'explique jamais rien $\gg($ p. 31, n.17).Il semble que ce soient les idées des dirigeants qui comptent avant tout.

Avec le chapitre 3, composé encore de commentaires de textes sur l'organisation interne du parti et ses rapports avec la base, tout le monde, de Lénine à Mao en passant par Staline et Gramsci, se voit soupçonné d' "idéalisme ". La conclusion de J.-M. Piotte à la fin de ces deux parties de son ouvrage se ramène à une vieille règle de prudence interprétative en matière politique : n'écoutez pas ce qu'ils disent, voyez ce qu'ils font (p. 51: «il faut juger la ligne idéologique à la lumière de la pratique politique effectivement suivie»; p. 71 : «la morale officielle doit elle-même être perçue à la lumière de l'agir réel des membres du parti »). Ceci ne peut cependant suffire qu'en partie à expliquer pourquoi l'auteur s'en tient aux idées et aux écrits, à leurs conséquences politiques et à leurs décalages par rapport aux pratiques, sans rapporter les uns et les autres à des contradictions historiques et à des conditionnements de classe, comme il prétendait le faire.

$\grave{A}$ ce point, un espoir reste au lecteur : peut-être va-t-on sortir des explications de textes pour éclairer les dynamiques 
sociales, les structures matérielles et les bases économiques, la conjoncture historique et les rapports de classes dans les pays dits "socialistes». Cet espoir n'est pas vraiment fondé. Le chapitre suivant s'engage dans le rituel commentaire : "Lénine, en s'inspirant ... etc., etc. »! Suit alors une nouvelle analyse des idées du fondateur de l'URSS, un peu plus historique, il faut le reconnaître, qui en retrace l'évolution au gré des développements politiques et la met en parallèle avec l'instauration et la croissance d'une domination bureaucratique. Ce chapitre est toutefois particulièrement faible. Il supporte mal la comparaison avec des synthèses existant sur le même sujet et aisément accessibles ${ }^{5}$. L'exposé, qui porte sur des textes et des faits bien connus et de nombreuses fois analysés, reste confus, hâtif et peu clair. Surtout, l'absence du type d'explication prônée par l'auteur lui-même se fait de plus en plus durement sentir: pourquoi cette société-ci, à ce stade-là de son développement, a-t-elle historiquement et sur la base de sa composition de classe, produit un régime de domination bureaucratique? Les analyses de J.-M. Piotte se contentent de montrer que, par ses prises de position, Lénine a été l'instrument, le responsable et la victime à la fois de la bureaucratisation. Cette dernière n'est pas davantage expliquée.

Bien que cela devienne monotone, il faut encore constater que la partie suivante n'apporte rien de nouveau, étant plutôt un exposé des opinions et des réactions de Lénine (et des manœuvres de Staline) face à la question des nationalités, qu'une analyse de ce facteur lui-même. Rien ne permet en particulier de savoir pourquoi J.-M. Piotte reconnaît une telle autonomie au facteur national malgré son «postulat» selon lequel la lutte des classes structure les contradictions historiques de tout phénomène social.

Le chapitre 6 est le grand chapitre théorique du livre. Il s'agit, pour J.-M. Piotte, de proposer une théorie sur la nature des pays de l'Est. Il s'en tient à ce sujet à la notion de bureaucratie : "faute d'un meilleur terme" (p. 20 ; cette

5. Connatitre Lénine, de Marcel Liebman (Éd. Marabout, 1976), par exemple, ou L'Héritage de Lénine, de François Fejtö (Le livre de poche " Pluriel », 1977), pour ne prendre que deux titres remarquables en français. 
restriction demeurera inexpliquée), il parle donc ici de "classe bureaucratique " et de "société bureaucratique ». Cette thèse est essentiellement descriptive. L'auteur ne l'établit pas par comparaison avec d'autres : la thèse anarchiste d'une dictature des intellectuels, la thèse libérale qui fait du collectivisme l'origine de la bureaucratie, la thèse de l'hégémonie étatique de Jean Dru, la thèse voisine de Djilas sur la "nouvelle classe ", la thèse de la restauration asiatique de Safarevic, etc. ${ }^{6}$. Les plus «bourgeois» des politologues sachant depuis des lustres que ces pays sont marqués par un caractère fortement bureaucratique, on ne peut voir de " marxiste " dans cette thèse que l'affirmation selon laquelle la bureaucratie constituerait une « classe », de surcroît dominante. Faute de plus amples précisions sur ce que peut être une classe définie non pas par sa place dans le procès de production économique mais par l'exercice d'une fonction de contrôle politique, cette différence demeure purement nominale. Pourquoi cette bureaucratie, «classe » ou non, exerce-t-elle le pouvoir dans ces pays? La seule réponse qu'on puisse soupçonner à ce stade de l'exposé (réponse indirectement confirmée par l'importance accordée auparavant à la question du rapport science/idéologie) est que le parti révolutionnaire une fois au pouvoir aurait lui-même fait le lit de cette domination à la faveur du dogmatisme de ses dirigeants. En effet, se croyant porteur de la science, de la compétence, en un mot du savoir vrai, le parti écrasa toute opposition et se constitua en seule source de toute décision, de toute autorité, de tout contrôle. Favorisant par là la sclérose de ses propres cadres ouvriers et leur associant des cadres bourgeois, le parti devenait par le fait même le lieu et l'agent de la formation d'une nouvelle classe sociale. L'instauration d'un nouveau pouvoir de classe s'effectue ainsi, la bureaucratie usurpant, sous couvert de scientificité et de compétence, le contrôle que les masses étaient en théorie censées exercer. Nulle part directement explicitée par J.-M. Piotte, cette thèse est cependant assez clairement présente dans la synthèse introductive du ch. 6 (p. 128-9). De plus, il n'y a jusque-là aucune autre explication ouvertement énoncée.

6. Pour un panorama succinct, assez représentatif, de diverses théories sur la nature des pays socialistes, voir : Lucio Pellicani, Gulag o utopia. Interpretazioni del comanismo, SugarCo, 1978. 
Si la théorie est bien que ce sont " des positions prises par le parti [qui] encouragèrent l'émergence de la bureaucratie » (p. 131), la démarche du livre s'éclaire en effet quelque peu. Le parti et Lénine en particulier ont eu le tort historique de se croire porteurs de la vérité, et c'est de cette erreur (renforcée par d'autres, sur la démocratie interne, sur les nationalités, etc., qu'elle permet et aggrave) que découle la suite des événements conduisant à la bureaucratisation autoritaire (et à la constitution des bureaucrates en classe dominante). On comprend bien qu'il était, dans cette hypothèse, normal d'aller chercher dans les écrits de Lénine et de ses successeurs les preuves et les raisons. Ce qu'on ne comprend vraiment plus du tout, c'est ce qu'il peut bien y avoir de "marxiste " (même d'un marxisme aussi " minoritaire " et " marginal " qu'on voudra) à attribuer à des erreurs théoriques et à leurs effets politiques un processus historique aussi fondamental que la formation même d'une nouvelle classe dominante. N'est-ce pas exactement un exemple de ce que lui-même baptise "une conception idéaliste de l'histoire" ("réduite à celle des idées », p. 10) ? Il raille Althusser, qui "expliquait les déformations des pays socialistes par une déviation idéologique : l'économisme » (p. 17). Sa position serait-elle moins «idéaliste » parce qu'elle identifie une autre déviation idéologique (le "scientisme ", ou peut-être tout simplement le dogmatisme) dans les conceptions de Lénine ainsi que d'autres erreurs adjuvantes (le « centralisme », etc.) ?

Je voudrais être bien clair sur ce point central. Mon propos n'est pas de reprocher à J.-M. Piotte d'être ou non "idéaliste ". Je prétends plutôt que son ouvrage est incohérent (ce qui est à mes yeux autrement plus grave). Que tout se joue pour lui au plan des conceptions ne peut rien expliquer d'autre que l'étrange démarche de son livre (succession de commentaires). Quelle consistance peut-on reconnaître à une pensée qui affirme qu'on doit tout expliquer par les luttes de classes et qui explique l'apparition même des classes en présence par les erreurs théoriques des dirigeants?

Bien sûr, il invoque quelquefois l'état social ou économique du pays, mais c'est seulement pour éclairer les motifs ou le contexte d'un changement de position chez tel penseur, ou 
pour indiquer les pratiques politiques fâcheuses qui en découlent, mais non pour les reconduire à un conditionnement par des forces sociales on infrastructurelles. Ce serait pourtant là, d'après ses propres principes, le seul type valable d'explication. Il existe d'ailleurs de telles tentatives, par exemple chez David Rousset (La Société éclatée, Paris, 1973), chez Ulf Wolters (Grundlagen des Stalinismus, Berlin, 1975), chez Marc Paillet (Marx contre Marx, Paris, 1971), chez Rudolf Bahro (L'Alternative, Paris, 1979) et beaucoup d'autres. Mais J.-M. Piotte semble les négliger délibérément. Il se réfere à une seule tradition d'analyse, celle qui va de Bruno Rizzi à Cornélius Castoriadis et Claude Lefort ${ }^{7}$. On peut d'ailleurs trouver aussi déplorable que son illogisme de base la superbe avec laquelle il ignore tout ce qui existe sur la question. Contrairement au conseil pertinent de $\mathrm{R}$. Linhart (dont il se réclame par ailleurs), il n'examine pas les travaux produits par des analystes ayant une connaissance directe et vécue des sociétés considérées $^{8}$, à part deux allusions fort peu probantes à Andras Hegedüs. De même, à l'exception de quelques articles de la revue Est-Ouest et de la Revue de l'Est évoqués en passant dans les derniers chapitres, il ignore tout des thèses non marxistes sur les problèmes de l'idéologie communiste et sur la nature des pays socialistes. Cette exclusion de principe indique qu'il n'a pas liquidé complètement en lui-même la croyance qu'il prétend combattre, selon laquelle le marxisme serait plus scientifique que les recherches idéologiques des philosophes et sociologues bourgeois ${ }^{9}$. . .

7. Si l'on comprend bien, ces deux derniers seraient l'exemple du " marxisme marginal " dont J -M. Piotte se réclame contre le « marxisme dominant". Ceci est d'autant moins éclairant que $\mathrm{C}$. Castoriadis se déclare antimarxiste depuis déjà des années

8. Et dont il aurait pu trouver, entre autres, un aperçu dans Roberto Gatti, $I$ marxismi all'opposizione nei paesi dell'est, Città Nuova, 1978 ; J. Kuron et K. Modzelewski, Il marxismo polacco all'opposizione, Samona e Savelli, 1967 ; Svetozar Stojanovitch, Between I. Jeals and Reality, Oxford U.P., 1973, (tr. it. : Gli ideali e la realtà, Feltrinelli, 1970); Milovan Djilas, La nouvelle classe dirigeante, 1975, Plon. De parution plus récente, il faut aussi signaler, outre le livre de R. Bahro déjà indiqué (mais dont une version anglaise circulait et dont les thèses avaient été amplement rapportées dans les revues et la presse française, italienne et anglaise), celui de G. Konràd et I. Szelényi, La marche au pouvoir des intellectuels, Le Seuil, 1979.

9. Naturellement, il n'est pas fait mention dans les «questions de philosophie » des critiques classiques du marxisme (Weber, Schumpeter, Popper, Kelsen, Russell, Von Mises, Von Hayek, de Man, Arendt, Aron, etc.). Pas question non plus de Monnerot (Sociologie du communisme), de Caillois (Description du marxisme), de Leff (The Tyranny of Concept), de Paillet (Le rêve et la raison). Ni trace de Louis Fischer (Du régime soviétique), 
Des idées nouvelles vont toutefois apparâttre soudainement d'ici la fin du volume. Nous devons donc réserver notre jugement final et aborder la septième partie. Outre l'inévitable commentaire du texte de Lénine, ce chapitre offre une série de discussions peu suivies où sont expéditivement rejetée la notion de «capitalisme d'État», nuancées les assimilations entre sociétés fascistes et socialistes, introduite sans autre forme de procès l'opinion que «capitalisme " bourgeois et "socialisme » bureaucratique ne seraient que «les deux grandes formations de l'actuel mode de production » (mais l'auteur nous réserve pour la fin ses quelques rares précisions sur ce "mode de production" inédit), affirmé que "les intellectuels» et «la bureaucratie " constituent en URSS "deux classes sociales" (sans que cette curieuse affirmation ne conduise l'auteur à nous expliquer quelles sont les diverses "classes " qu'on y trouve d'après lui) ${ }^{10}$.

Quand au chapitre 8, il aborde la question des rapports économiques de l'URSS avec ses "alliés", puis celle de la compétition mondiale entre bourgeoisie et bureaucratie. Il en conclut prudemment que les facteurs politiques l'emportent sur les facteurs économiques; que l'issue de la lutte internationale est imprévisible; que les bureaucraties occidentales ne semblent pas pouvoir se substituer de l'intérieur à leurs bourgeoisies. Quant à savoir si les bureaucraties occidentales constituent elles aussi une "classe sociale " et si cette " classe " éventuelle est la même que celle dont il est question

Merle Fainsod (Comment l'URSS est gouvernée), John Plamenatz (German Marxism and Russian Bolshevism), J.P. Nettl, (Bilan de l'URSS), Rostow et Lewin (The Dynamics of Soviet Society), Barrington Moore (Terreur et progrès en URSS), L.G. Churchward (Contemporary Soviet Government), Pierre Sorlin (La société soviétique), Antonio Carlo (La natura sociale dell'URSS), Mc Closky et Turner (The Soviet Dictatorship), Carew Hunt (Théorie et pratique du communisme), Rene Ahlberg (Die Sozialistische Bürokratie; trad. it., La Burocrazia sorialista), Max Schachtman (The Bureaucratic Revolution), V. Pérez Diaz (Estado, Burocracia y Sociedad Civil ; en angl. State, Bureaucracy and Civil Society), etc. L'auteur se souvient-il aussi de Merleau-Ponty (Les aventures de la dialectique), de Victor Serge (Destin diune révolution), de Pierre Hervé (La révolution et les fétiches), de Clavel (Qui est aliéné ?) ? On ne sait s'il faut s'émerveiller ou s'attrister de trouver, chez un professeur de science politique à l'université une telle innocence intellectuelle et historique. Se réveillant soudain de son sommeil léniniste, le savant découvre ex nibilo, en 1979, la vraie nature du socialisme et annonce sans fausse modestie sa trouvaille: l'URSS est bureaucratique. Foin des prédécesseurs ! Quelle époque riche en surprises, tout de même !

10. Plus loin, en p. 170 , il évoque soudain : la classe ouvrière, la paysannerie, les employés et les intellectuels. Plus la bureaucratie, bien entendu. 
à propos des pays de l'Est, J.-M. Piotte évidemment n'en souffle mot.

Dans sa conclusion générale, il avance que seule une large alliance du peuple « (ouvriers, paysans, employés) avec une fraction des intellectuels semble être une condition nécessaire pour une lutte efficace, voire victorieuse, contre la bureaucratie $\gg$ (p. 171). Une telle perspicacité déjoue la critique, on le reconnaîtra avec moi. Il énonce alors une nouvelle version, plus complète, de sa "théorie "sur la nature des sociétés "socialistes »: l'alliance des cadres du parti et des spécialistes d'origine bourgeoise sous Lénine a pu fonder, malgré l'abolition de la propriété privée, la domination d'une nouvelle classe grâce à un rapport d'appropriation ${ }^{11}$. L'objectif des luttes actuelles est défini par lui sur cette base: il s'agira d'une version du communisme qui, enrichie de cette expérience, veillera à supprimer non seulement la propriété privée des moyens de production, mais aussi l'appropriation bureaucratique et les divisions qu'elle suppose (intellectuel/manuel, dirigeants/dirigés, dominants/dominés).

C'est à ce point qu'il s'aventure à nous éclairer enfin sur son «mode de production actuel » (commun aux sociétés capitalistes et socialistes). Il le définit par l'organisation du travail, fondée sur " le rapport d'appropriation par lequel la société se constitue sur la division entre la force de travail et ceux qui l'administrent, l'organisent, la dirigent et la dominent » (p. 172). Par un raisonnement d'une parfaite circularité, J.-M. Piotte explique ainsi la domination d'une classe (bureaucratie ou bourgeoisie) sur les autres par le fait que la structure du « mode de production actuel » se caractérise par une division entre dominants et dominés. D'une part, en effet, il affirme (p. 172-3) que « l'actuel mode de production repose sur un développement sans précédent des forces productives qui suppose un rapport de production, ou, plus précisément, un rapport d'appropriation où les travailleurs sont séparés de leurs moyens de production » (par la taylorisation, la parcellisation, la mécanisation du travail). D'autre part, cette « orga-

11. Il signale chez Castoriadis et Balibar des distinctions entre propriété juridique, propriété réelle, possession et appropiation. Comme d'habitude, cette question fondamentale et complexe est réglée en quelques lignes peu explicites. 
nisation du travail » implique une classe d'organisateurs, rendant, dit-il, « nécessaire une force sociale qui divise, répartit, et organise le travail, une force sociale qui planifie» non seulement les choses mais les hommes (p. 173).

Il est évidemment permis de douter, face à cette tentative de théorisation in extremis, que la notion de «mode de production " conserve à ce niveau de généralité une quelconque vertu explicative. Si le mode de production «actuel » en question se définit et se caractérise par un rapport quasi structurel de division entre travail et organisation, rien d'étonnant en tout cas à ce qu'il rende nécessaire et explique l'appropriation qu'effectue la classe des organisateurs aux dépens des organisés. S'il en est ainsi, on voit cependant encore plus mal qu'avant ce que les erreurs doctrinales de Lénine pouvaient bien avoir comme rôle déterminant dans la bureaucratisation. On se demande aussi pourquoi J.-M. Piotte n'explique pas tout le devenir des révolutions socialistes par la thèse de la modernisation défensive ${ }^{12}$.

Quoi qu'il en soit de la justesse et de la profondeur de cette théorie d'un «mode de production actuel », il faut admettre que l'objectif politique de lutte contre la division entre organisateurs et organisés en découle tout naturellement. Cogestion, participation, "enrichissement des tâches ", travail reconstitué par petites équipes, en Occident, autogestion yougoslave, lutte contre la séparation travail manuel/travail intellectuel durant la révolution culturelle en Chine, sont ici les références prévisibles. J.-M. Piotte aboutit cependant à une analyse plutôt pessimiste de la conjoncture. L'efficacité productive du "mode de production " régnant empêche tout retour en arrière, vu l'adhésion des masses populaires, dont les besoins matériels demeurent insatisfaits, au modèle du développement économique (p. 174). La lutte politique révolutionnaire elle-même, exigeant un minimum d'organisation, risque de renforcer la division dirigeants/dirigés. Dans un raisonnement de quelques lignes, l'ouvrage s'achève alors elliptiquement par deux propositions qui semblent contradictoires. La première est que le rapport d'appropriation que

12. Cf. L. Pellicani, op. cit., p. 79-86. 
provoque $^{13}$ cette division entre dominants et dominés dans le «mode de production actuel » n'est " ni nécessaire ni éternel » (p. 176). La seconde est que les fonctions de gestion et d'organisation remplies par la classe bureaucratique ne pouvant être exercées directement par la classe ouvrière, d'une part, et la prise du pouvoir d'État par des forces révolutionnaires conduisant à la reproduction d'un semblable rapport d'appropriation, d'autre part, « les forces révolutionnaires doivent donc lutter pour exercer un contrôle sur la bureaucratie, viser à dominer, de l'extérieur, les appareils bureaucratiques, lutte permanente qui peut sans doute nous rapprocher du communisme, sans que nous le réalisions jamais, le communisme ne devenant qu'une idée limite qui oriente le sens des luttes des opprimés» (p. 177). Un auteur qui avait commencé en dénonçant la position des « nouveaux philosophes », affirmant qu'il y aurait toujours un Pouvoir et qu'il fallait s'en tenir à une Résistance perpétuelle qui le contienne, rejoint finalement une thèse voisine.

En résumé, cet ouvrage n'a que des défauts. Comme une telle constation pourrait laisser supposer que je me livre à un règlement de comptes politique, je voudrais que l'on me comprenne bien. Je fais à Marxisme et pays socialistes plusieurs types de reproches. Un premier est l'extrême banalité et le caractère peu élaboré et peu critique de ses thèses principales (les « erreurs » de Lénine et de ses successeurs ont conduit les pays socialistes à un régime bureaucratique oppressif ; les structures de production des sociétés industrielles favorisent un renforcement du pouvoir de la techno-bureaucratie sur les masses). Un second est son incohérence interne (par exemple lorsqu'il affirme que les faits sociaux s'expliquent par la lutte des classes, mais attribue à des positions théoriques la formation même d'une classe clé). Un troisième est sa très grande faiblesse d'argumentation et la médiocrité de son exposition. Un quatrième est la désinvolture avec laquelle il néglige d'examiner et de critiquer les nombreuses théories existant sur les mêmes sujets. Un cinquième est le caractère bâclé et ambigu de sa prise de position finale.

13. Ou : qui provoque. On ne sait plus trop. 
Tout ceci ne traduit aucunement de ma part un désaccord avec l'esprit général dont on peut supposer qu'il a conduit J.-M. Piotte à rédiger ces pages. En effet, je suis de ceux qui pensent depuis longtemps que la distinction althussérienne science/idéologie est absurde appliquée au marxisme, qui est une philosophie et une idéologie politique. Je suis également de ceux qui croient que les versions répandues du marxismeléninisme orthodoxe ont fait faillite et qu'il faut au minimum s'affirmer révisionniste si l'on veut sauver le peu de chances historiques qui restent à un progressisme social non autoritai$\mathrm{re}^{14}$. Bien que je ne sois pas absolument convaincu qu'il existe vraiment un «savoir marxiste» ayant fait ses preuves dans l'explication du capitalisme, je suis moi aussi porté à croire que des perspectives ou une approche d'inspiration marxiste (déterminisme par les infrastructures, analyse politique en termes de conflits entre classes sociales, critique symptomale des idéologies officielles) doivent être utilisées à volonté pour élucider l'histoire mystifiée des pays "socialistes ${ }^{15}$. Je ne doute pas non plus que les paroles et les actes de Lénine permettent de lui attribuer une large responsabilité historique dans le processus de retournement d'idéaux positifs en réalités négatives qui a marqué les années postrévolutionnaires en URSS (bien que cette façon de poser le problème me paraisse partielle et insuffisante). Je ne rejette pas non plus (ce serait difficile) l'idée selon laquelle la bureaucratie est un facteur social caractéristique et déterminant dans les sociétés de type soviétique. Je crois simplement que la reconnaissance de ce fait nous laisse très loin d'avoir mené à bien l'immense travail théorique qui consistera à rendre compte de la «nature sociale » des pays de l'Est. Je suis enfin porté à accepter la thèse qui veut qu'une révolution politique globale comme celle que prône la tradition léniniste soit actuellement exclue dans les sociétés industrielles avancées à démocratie parlementaire

14. Cf., par exemple, la tentarive de M. Albert et R. Hahnel, Unortbodox Marxism, South End Press, 1978. Il faut aussi relire Bernstein (Les présupposés du socialisme, Le Seuil, 1974). Paul Mattick nous rappelle une tradition trop souvent occultée dans Anti-Bolshevik Communism, M.E. Sharpe, 1978.

15. Ne serait-ce que pour des raisons d'efficacité tactique, qu'illustre l'exemple de $\mathbf{R}$. Bahro : ce sont les attaques portées de l'intérieur, au nom même du marxisme, qui sont les plus insupportables à l'idéocratie. On le savait depuis Luther. 
(et qu'il nous faut en conséquence réinventer un autre progressisme).

Mais cette large base d'accord et ces éléments incontestables de parenté d'idées n'ont pu que me rendre plus aiguë la pénible évidence que Marxisme et pays socialistes est un fort méchant livre, indigne de son auteur.

\section{Collège Ahuntsic}

P.-S. : Sur le fond de la " Note " qui suit, j’ai été frappé par son énoncé central. Deux constatations de base, qui ne m'avaient pas échappé : « le prolétariat n'a pu consolider son pouvoir " et "les tâches d'organisation ont suscité la formation d'une nouvelle classe dominante ". Bien. C'est ici naturellement que commence le vrai problème théorique et politique : pourquoi en a-t-il été ainsi ? Partie positive de la réponse: "le léninisme a justifié certaines mesures qui ont favorisé l'émergence et la domination de la bureaucratie". C'est ce que i'avais cru comprendre. Partie négative de la réponse : "il serait erroné d'y chercher la cause ou le responsable historique de cette nouvelle domination de classe. "Fort bien encore. C'est même exactement mon avis. Mais alors, quelle est à la fin cette cause ? C'est à cette question que j'ai en vain cherché une réponse dans le livre. La note a beau dénoncer mon simplisme et nous inviter à une lecture plus attentive, plus approfondie et plus politique, elle ne juge pas utile de dire un seul mot de cette réponse clé qui ne vient jamais. Comprenne qui pourra. (Bien sûr, il faut peut-être entendre ceci : c'est la nécessité de l'organisation qui provoque l'apparition d'une classe dirigeante. Mais alors pourquoi refuser de tenir compte des critiques classiques et libérales du marxisme, qui n'ont jamais cessé de dire précisément la même chose ? Comment peut-on soutenir une telle thèse et se croire encore " narxiste»? Je vois là, sans éclectisme ni prudence, sans malveillance ni malhonnêteté, une grande confusion. C'est tout.)

L. -M. V 